\title{
Relative Performance Evaluation In a Multi-Plant Firm*
}

\author{
Annalisa Luporini \\ Department of Economics, University of Florence, \\ via delle Pandette 9, 50127, Firenze, Italy \\ (email: luporini@unifi.it)
}

January 2005

\begin{abstract}
We analyze optimal compensation schedules for the directors of two plants belonging to the same owner and producing the same good but serving geographically differentiated markets. Since the outcome of each director depends on his own effort and on a random variable representing market conditions, the problem takes the form of a principal multi-agent model. We first provide appropriate extensions of the MLR and CDF conditions that ensure the validity of the first-order approach in the single agent case. Then, we show that affiliation of the random variables is a necessary and sufficient condition for the compensation of one director to negatively and monotonically depend on the performance of the other.

Keywords: Principal-agent problems, relative performance evaluation, first-order approach, monotone likelihood ratio, affiliation.
\end{abstract}

JEL code: D23, D82

\section{Introduction}

Relative performance evaluation (RPE) is a common practice in organizations. Comparisons among the performances of different agents who are assigned to similar tasks are sometimes explicitly contained in labor contracts. Sellers' compensations usually depend on the level of sales relative to the achievements of other sellers; CEOs compensations may contain a bonus based on a comparison with competitors' returns. In other cases, even in the absence of explicit contract clauses, compensations implicitly depend on the results of peers working in similar conditions. Informal comparisons among workers are crucial both when firms set internal performance standards and when hierarchical superiors evaluate their subordinates.

*I thank Ray Rees for helpful comments. 
Agency theory (Holmström, 1982; Mookherjee, 1984) has provided a rationale for these practices in terms of their informational content. When agents' outcomes are subject to a common element of uncertainty, the output of each individual acts both as a signal of his own performance and as a signal of the realizations of the common uncertain parameter. As a consequence, comparisons of agents' performances are valuable because they bring additional pieces of information to the system. This has important consequences also for the design of both jobs and accounting systems. Duplication of tasks or job rotation may be of value to the firm as well as the definition of comparable profit/cost centers precisely because they allow relative evaluation.

Here we consider a firm producing a single good in two plants located in geographically different areas. Each plant faces identical costs but serves a different market whose stochastic demand is positively related to that of the other market. Such a positive relation might represent common macro factors that add to idiosyncratic demand components. These market conditions are reflected in stochastic and positively dependent returns to the (unobservable) effort of each plant's director. We investigate the form of the compensation schedules that the owner of the firm (the principal) should offer to directors (the agents) in order to motivate them to exert the optimal level of effort. Given that returns are stochastically dependent, we expect that optimal compensation schedules will be interdependent. In particular we want to investigate the sign of such interdependence and to determine conditions that ensure monotonically negative relation between the compensation of one director and the performance of his colleague.

Although the informational relevance of relative performance evaluation has been stressed in the literature ${ }^{1}$, the model with one principal and many agents has not been completely characterized. First of all, the applicability of the firstorder approach (i.e. of the procedure that substitutes incentive compatibility constraints with their first-order conditions) has not been investigated. Extensions of the monotone likelihood (MLR) and of the convexity of the distribution function $(\mathrm{CDF})$ conditions that ensure the validity of the first-order approach in the single agent case (Mirrlees, 1979; Rogerson, 1985) have only been established for the general multi-signal case (Sinclair-Desgagné, 1994). Our multi-agent model can be considered part of the class of multi-signal models but has some specificities. In multi-signal models, several signals are observed concerning a single level of effort. In our model, with many agents and separable production functions, each agent's output is a signal both of that agent's effort and

\footnotetext{
${ }^{1}$ Following the theoretical results of Holmström (1982) and Mookherjee (1984) there have also been several attempts to verify the empirical relevance of relative performance evaluation in CEO compensations. This literature has focussed on the implicit (supposedly negative) relation between total CEO pay and market/sector performance. Results are mixed. Antle and Smith (1986) and Janakiraman Lambert and Larcker (1992), performing longitudinal analyses, conclude that evidence is only weakly consistent with RPE. Gibbons and Murphy (1990), analysing pooled cross-sectional time series, find a negative and significant relation between changes in CEO compensations and both market and industry performance. Recently, Kren (2002) has verified that the use of RPE is related to the level of common uncertainty thus providing a closer test of agency theory.
} 
of the realization of the random variables affecting the output of other agents. However it is not a direct signal of their efforts. Due to this peculiarity, the extension of the first-order approach is somewhat simplified. Secondly, the form of the dependence of an individual's compensation on others' performance has not been thoroughly studied. Holmström and Milgrom (1990) analyze the form of optimal compensations in a linear model with normally distributed random variables and exponential utility functions. They show that positive (negative) correlation implies negative (positive) dependence of the compensation of one agent on the performance of his co-workers. No analogous result, however, has been proved in more general settings.

After presenting the model (section 2), we investigate the question of the validity of the first-order approach in our multi-agent setting (section 3). We show that the extensions of the MLR and CDF conditions take a slightly different form and that fewer assumptions are needed than in the general multi-signal model. We then point out (section 4) that affiliation of the random variables affecting the directors' outcomes is both necessary and sufficient for the salary of each director to be nonincreasing in the performance of his colleague. We thus show that positive dependence of the random variables implies negative dependence of individual compensations on the results of the other agents. Section 5 concludes.

\section{The model}

Consider a firm consisting of two plants, $A$ and $B$, each run by a different director (agent). The two plants belong to the same owner (principal) and produce the same good which is sold in geographically separate but stochastically dependent markets. The monetary outcome of each plant $x_{i}$ is a function of the unobservable effort of director $i, a_{i}$, and of the random variable $\theta_{i}$ representing market conditions and all other factors that affect $x_{i}$ and that are not controlled by director $i$. Formally $x_{i}=x_{i}\left(a_{i}, \theta_{i}\right), i=A, B$, with $a_{i} \in A_{i}=\left(\underline{a}_{i}, \bar{a}_{i}\right) \subset R^{+}$ and $\theta_{i} \in \Theta_{i}=\left(\underline{\theta}_{i}, \bar{\theta}_{i}\right) \subseteq R$. The range of $x_{i}, X_{i}=\left(\underline{x}_{i}, \bar{x}_{i}\right)$, is invariant with respect to $a_{i}$. Moreover we assume that the outcome is increasing in both the director's effort, $\frac{\partial x_{i}}{\partial a_{i}}>0$, and in the realization of the random variable, $\frac{\partial x_{i}}{\partial \theta_{i}}>0$. The effect of $\theta_{i}$ on the marginal product is also (weakly) positive $\frac{\partial^{2} x_{i}}{\partial a_{i} \partial \theta_{i}} \geq 0$. As a consequence the inverse functions $x_{i}^{-1}\left(a_{i}, x_{i}\right)$ are well defined at each $a_{i} \in A_{i}$ with derivatives: $\frac{\partial x_{i}^{-1}}{\partial a_{i}}<0, \frac{\partial x_{i}^{-1}}{\partial x_{i}}>0, \frac{\partial^{2} x_{i}^{-1}}{\partial a_{i} \partial x_{i}} \leq 0$. Let $x$, $a$, and $\theta$ denote vectors of outcomes, efforts and random variables respectively. The $\theta_{i}$ have joint density $g(\theta)$ and are affiliated dependent random variables. Affiliation, which is discussed extensively in section 4 , is meant to capture the positive dependence between market conditions faced by the two plants. The realizations of $\theta$ are not observable but $g(\theta)$ is common knowledge.

Since Mirrlees (1974), it has become common practice to analyze such an 
agency problem by suppressing the $\theta_{i}$ and considering the outcomes $x_{i}$ directly as random variables parametrized by the effort levels $a_{i}$. We will follow this approach but we will keep in mind the original setting since it is crucial for the proof of our result. Let then $f(x ; a)$ be the joint density function obtained as a transformation of $g(\theta)$ via the functions $x_{i}$, i.e. $f(x ; a)=g\left(x^{-1}\right) \frac{\partial x_{1}^{-1}}{\partial x_{1}} \frac{\partial x_{2}^{-1}}{\partial x_{2}}$.

The directors have additively separable utility functions over income and effort, $u_{i}\left(y_{i}\right)-c_{i}\left(a_{i}\right)$, with $u_{i}$ concave and continuously differentiable and $c_{i}$ convex and twice continuously differentiable. The monetary outcome $x_{i}$ accrues to the risk neutral owner who has to compensate director $i$ with, possibly contingent, salary $y_{i}(x)$. The owner wants to maximize his expected profit, given that the agents choose the effort levels so as to maximize their expected utility, and that they receive at least their reservation level of utility $\bar{U}_{i}$. In other terms, the owner has to solve:

$$
\max _{a_{i}, y_{i}} \sum_{i} \int\left(x_{i}-y_{i}(x)\right) f(x ; a) d x, \quad i=A, B
$$

subject to:

$$
\begin{gathered}
\int\left[u_{i}\left(y_{i}(x)\right)-c_{i}\left(a_{i}\right)\right] f(x ; a) d x \geq \bar{U}_{i} \quad i=A, B, \\
a_{i} \in \underset{\widehat{a}_{i}}{\arg \max } \int\left[u_{i}\left(y_{i}(x)\right)-c_{i}\left(\widehat{a}_{i}\right)\right] f(x ; a) d x \quad i=A, B .
\end{gathered}
$$

Note that incentive compatibility constraints (3) impose that $a_{i}$ be a Nash equilibrium of the game played by the directors. In other words we are assuming that the agents act non cooperatively, i.e. that they do not engage in collusive behavior. This seems reasonable in the present context where directors do not observe each other's effort which makes the striking and enforcing of a covert contingent side contract quite problematic. ${ }^{2}$

\section{A first-order approach formulation of the prob- lem}

Under the first-order approach, incentive compatibility constraints (3) are replaced by their first-order conditions. However, to be allowed to do so, we need to ensure that the expected utility of the agents are concave in $a_{i}$. It is well known that in the standard single agent case the validity of the first-order approach is guaranteed by the monotone likelihood ratio (MLR) together with the convexity of the distribution function (CDF) assumptions ${ }^{3}$. We will now provide extensions of these assumptions for our two-agent case.

\footnotetext{
${ }^{2}$ Side contracting in principal multi-agent models is analyzed in Itoh (1993) and Holmström and Milgrom (1990).

${ }^{3}$ See Mirrlees (1979) and Rogerson (1985). Subsequently Jewitt (1988) has proposed alternative conditions to the $\mathrm{CDF}$.
} 
Note that, if substituted by its first-order conditions, (3) becomes:

$$
\int u_{i}\left(y_{i}(x)\right) f_{a_{i}}(x ; a) d x-c_{i}^{\prime}\left(a_{i}\right)=0 \quad i=A, B .
$$

Solving (1) subject to (2) and (4), the Kuhn-Tucker conditions on $y_{i}(x)$ give:

$$
\frac{1}{u_{i}^{\prime}\left(y_{i}(x)\right)}=\lambda_{i}+\mu_{i} \frac{f_{a_{i}}(x ; a)}{f(x ; a)} \quad i=A, B
$$

where $\lambda_{i}$ and $\mu_{i}$ are the multipliers associated with participation and incentive compatibility constraints (2) and (4) respectively. This form is particularly convenient in that it directly relates the shape of the compensation schedule $y_{i}(x)$ to the shape of the ratio $\frac{f_{a_{i}}(x ; a)}{f(x ; a)}$.

In order to justify this procedure let us first make the following assumption:

Partial monotone likelihood ratio condition (PMLRC): $\frac{f\left(x_{i}, x_{k} ; a_{i}^{+}, a_{k}\right)}{f\left(x_{i}, x_{k} ; a_{i}^{-}, a_{k}\right)}$ is nondecreasing in $x_{i}$ for $a_{i}^{+} \geq a_{i}^{-}, i, k=A, B, i \neq k$.

PMLRC means that a higher $x_{i}$ is a better signal for a higher $a_{i}$, at each $\left(x_{k} ; a_{k}\right)$, i.e. that it is more likely to have a higher $x_{i}$ if $a_{i}$ is large independently of what the other director has done. This seems the natural extension of the MLR condition of the single agent case to our multi-agent setting: we simply assume that MLR holds at any given couple $\left(x_{k}, a_{k}\right)$. The form of such extension marks a first difference from the general multi-signal model in which (contrary to our case where, given $a_{k}, x_{k}$ is a signal on $\theta_{i}$ and not on $a_{i}$ ) there is an entire vector of signals correlated to the effort of one agent and where the MLR condition is then defined with respect to the entire vector (Sinclair-Desgagné, 1994).

Lemma 1: PMLRC implies that $\frac{f_{a_{i}}(x ; a)}{f(x ; a)}$ is non decreasing in $x_{i}, \quad i=A, B$.

Proof: The proof follows exactly the proof given in Milgrom's (1981) Proposition 5 for the single agent case. $\square$

This together with (5), in turn implies

Lemma 2: PMLRC implies $\mu_{i}>0$ and is a necessary and sufficient condition for $y_{i}(x)$ to be nondecreasing in $x_{i}$.

Proof: The proof follows the standard argument of the corresponding proof for the single agent case.

This is just a straightforward extension of a well-known result in single agent literature. To ensure a positive relation between the compensation of a director and his own outcome we have to assume monotonicity of the likelihood ratio. 
PMLRC, however, is not sufficient to ensure the validity of the first-order approach. In order to be able to extend also the CDF condition, let us then define $H_{i}\left(x_{i}, x_{k} ; a_{i}, a_{k}\right) \equiv \int_{\underline{x}_{i}}^{x_{i}} f\left(\widehat{x}_{i}, x_{k} ; a_{i}, a_{k}\right) d \widehat{x}_{i}$, the cumulative distribution of $x_{i}$ at $x_{k}$ and $a_{k}$. We can then use an argument similar to that of Milgrom's (1981) Proposition 1 to prove the following Lemma.

Lemma 3: From PMLRC it follows that $H_{i}\left(x_{i}, x_{k} ; a_{i}^{+}, a_{k}\right) \leq H_{i}\left(x_{i}, x_{k} ; a_{i}^{-}, a_{k}\right)$ for $a_{i}^{+} \geq a_{i}^{-}$. The distribution parametrized by $a_{i}^{+}$first-order stochastically dominates that parametrized by $a_{i}^{-}$.

Proof: Consider $a_{i}^{+} \geq a_{i}^{-}, i, k=A, B, \quad i \neq k$ and fix $\underline{x}_{i}<x_{i}^{*}<\bar{x}_{i}$. For $x_{i} \leq x_{i}^{*}$ PMLRC implies:

$$
\frac{\int_{\widetilde{x}_{i}>x_{i}^{*}} f\left(\widetilde{x}_{i}, x_{k} ; a_{i}^{+}, a_{k}\right) d \widetilde{x}_{i}}{f\left(x_{i}, x_{k} ; a_{i}^{+}, a_{k}\right)} \geq \frac{\int_{\widetilde{x}_{i}>x_{i}^{*}} f\left(\widetilde{x}_{i}, x_{k} ; a_{i}^{-}, a_{k}\right) d \widetilde{x}_{i}}{f\left(x_{i}, x_{k} ; a_{i}^{-}, a_{k}\right)}
$$

or

$$
\frac{f\left(x_{i}, x_{k} ; a_{i}^{+}, a_{k}\right)}{\int_{\widetilde{x}_{i}>x_{i}^{*}} f\left(\widetilde{x}_{i}, x_{k} ; a_{i}^{+}, a_{k}\right) d \widetilde{x}_{i}} \leq \frac{f\left(x_{i}, x_{k} ; a_{i}^{-}, a_{k}\right)}{\int_{\widetilde{x}_{i}>x_{i}^{*}} f\left(\widetilde{x}_{i}, x_{k} ; a_{i}^{-}, a_{k}\right) d \widetilde{x}_{i}} .
$$

Integrating the above expression over $x_{i} \leq x_{i}^{*}$ yields:

$$
\frac{H_{i}\left(x_{i}^{*}, x_{k} ; a_{i}^{+}, a_{k}\right)}{1-H_{i}\left(x_{i}^{*}, x_{k} ; a_{i}^{+}, a_{k}\right)} \leq \frac{H_{i}\left(x_{i}^{*}, x_{k} ; a_{i}^{-}, a_{k}\right)}{1-H_{i}\left(x_{i}^{*}, x_{k} ; a_{i}^{-}, a_{k}\right)}
$$

which implies $H_{i}\left(x_{i}, x_{k} ; a_{i}^{+}, a_{k}\right) \leq H_{i}\left(x_{i}, x_{k} ; a_{i}^{-}, a_{k}\right)$ for $a_{i}^{+} \geq a_{i}^{-}$

Since $y_{i}(x)$ is nondecreasing in $x_{i}$ this implies that the expected utility of director $i, \int u_{i}\left(y_{i}(x)\right) f(x ; a) d x$, is increasing in $a_{i}$. To ensure that it does so at a decreasing rate we also make the following assumption.

Partial convexity of the distribution function condition (PCDFC): $H_{i}\left(x_{i}, x_{k} ; a_{i}, a_{k}\right)$ is convex in $a_{i}$.

We are then in a position to prove:

Proposition 1. Under PMLRC and PCDFC agent i's expected utility is concave in $a_{i}$.

Proof. Integrating $\int u_{i}\left(y_{i}(x)\right) f(x ; a) d x$ by parts with respect to $x_{i}$, we obtain:

$$
\begin{aligned}
& \int_{X_{k}} u_{i}\left(y_{i}\left(\bar{x}_{i}, x_{k}\right)\right) H_{i}\left(\bar{x}_{i}, x_{k} ; a_{i}, a_{k}\right) d x_{k}- \\
& \int_{X_{k}}\left[\int_{X_{i}} u_{i}^{\prime}\left(y_{i}\left(x_{i}, x_{k}\right)\right) \frac{\partial y_{i}\left(x_{i}, x_{k}\right)}{\partial x_{i}} H_{i}\left(x_{i}, x_{k} ; a_{i}, a_{k}\right) d x_{i}\right] d x_{k}
\end{aligned}
$$


where $y_{i}\left(\bar{x}_{i}, x_{k}\right)=\lim _{x_{i} \rightarrow \bar{x}_{i}} y_{i}\left(x_{i}, x_{k}\right)$. The first term is a sum of constants with respect to $a_{i}$ while the second one is the sum of terms that are convex in $a_{i}$ multiplied by positive terms. Since $c_{i}\left(a_{i}\right)$ is convex, the expected utility of agent $i$ is concave in $a_{i}$.

Therefore the first-order approach is valid and (5) gives the optimal compensation $y_{i}(x)$. Our line of proof has paralleled that of the multi-signal model of Sinclair-Desgagné (1994). Note however that Lemma 3 is not valid in that context where the MLR condition is defined with respect to a vector of signals on the effort of one agent. This is the reason why Sinclair-Desgagné has to assume stochastic dominance of the cumulative distribution function of one signal.

\section{Optimal compensations}

From lemma 2, we know that the compensation $y_{i}(x)$ of director $i$ is nondecreasing in his own outcome $x_{i}$. Moreover, since the $\theta_{i}$ are not independent, we know (from Holmström, 1982) that $y_{i}(x)$ also depends on director $k$ 's result, $x_{k} \cdot{ }^{4}$ We do not know anything, however, about the sign of such relation. The incentive content of the positive link between director $i$ 's compensation and his own level of output is obvious: director $i$ will be motivated to exert a high level of effort in efficiently producing and selling the good. Note, however, the relevance of the MLR assumption for this result. Due to the informational content of level of outcome, in the absence of the MLR assumption, $i$ 's optimal compensation need not be monotonically increasing in $i$ 's own outcome. For example, the distribution of $x_{i}$ could be such that, up to a given level of outcome $\widehat{x}_{i}$, a higher $x_{i}$ signals a high level of effort, while for $x_{i} \geq \widehat{x}_{i}$, the level of outcome is related to high realizations of the random variable representing market conditions, independently of the effort exerted. In that case, for any give $x_{k}$, the optimal $y_{i}$ would be increasing up to $\widehat{x}_{i}$ and decreasing at $\widehat{x}_{i}$. Something similar might happen in the relationship between the optimal compensation of director $i$ and the outcome of director $k$ (depending on the form of the joint distribution of $x_{i}$ and $x_{k}$ ), if relatively higher levels of $x_{k}$ were to signal relatively higher values of $\theta_{i}$ up to $\widehat{x}_{k}$ but relatively lower values of $\theta_{i}$ for $x_{k} \geq \widehat{x}_{k}$. For any given $x_{i}$, director $i$ 's compensation would first decrease and then increase in $x_{k}$. Since we want to represent a situation in which there is positive stochastic dependence between the market conditions faced by the two directors, we want $y_{i}(x)$ to be monotonic in $x_{k}$. We now show that affiliation is the appropriate assumption to rule out non monotonicity.

Formally, a bivariate random variable $\theta=\left(\theta_{i}, \theta_{k}\right)$ is said to be affiliated (Milgrom and Weber, 1982; Tong, 1980) if:

$$
g\left(\theta_{i}^{+}, \theta_{k}^{+}\right) g\left(\theta_{i}^{-}, \theta_{k}^{-}\right) \geq g\left(\theta_{i}^{+}, \theta_{k}^{-}\right) g\left(\theta_{i}^{-}, \theta_{k}^{+}\right) \quad \text { for all } \theta_{i}^{+}>\theta_{i}^{-} \text {and } \theta_{k}^{+}>\theta_{k}^{-} \text {. }
$$

\footnotetext{
${ }^{4}$ Holmström shows that, unless the $\theta_{i}$ are independent, $\frac{f_{a_{i}}(x ; a)}{f(x ; a)}$ depends on the whole vector $x$, thus implying that $i^{\prime}$ s compensation depends on $x_{k}$ (see (5) above).
} 
Affiliation broadly means that the random variables tend to move together, i.e. that it is more likely to have a high realization of $\theta_{i}$ when there is a high rather than a low realization of $\theta_{k}$. It is thus a form of positive dependence. ${ }^{5}$

Proposition 2: Affiliation between the $\theta_{i} i=A, B$, is a necessary and sufficient condition for the compensation of director $i, y_{i}(x)$ to be a nonincreasing function of the outcome of director $k, x_{k}, i, k=A, B, i \neq k$.

Proof: Consider (5) for agent $i$. We can write:

$$
\frac{f_{a_{i}}\left(x_{i}, x_{k} ; a_{i}, a_{k}\right)}{f\left(x_{i}, x_{k} ; a_{i}, a_{k}\right)}=\frac{\partial g\left[x_{i}^{-1}\left(a_{i} ; x_{i}\right), x_{k}^{-1}\left(a_{k}, x_{k}\right)\right] / \partial x_{i}^{-1}}{g\left[x_{i}^{-1}\left(a_{i} ; x_{i}\right), x_{k}^{-1}\left(a_{k}, x_{k}\right)\right]} \frac{\partial x_{i}^{-1}}{\partial a_{i}}+\frac{\frac{\partial^{2} x_{i}^{-1}}{\partial x_{i} \partial a_{i}}}{\frac{\partial x_{i}^{-1}}{\partial x_{i}}}
$$

where $\frac{\partial x_{i}^{-1}}{\partial a_{i}}<0, \frac{\partial x_{i}^{-1}}{\partial x_{i}}>0, \frac{\partial^{2} x_{i}^{-1}}{\partial x_{i} \partial a_{i}} \leq 0$.

Note that for any $\theta_{i}^{+}$and $\theta_{i}^{-}, \frac{g\left(\theta_{i}^{+}, \theta_{k}\right)}{g\left(\theta_{i}^{-}, \theta_{k}\right)}=\exp \left[\int_{\theta_{i}^{-}}^{\theta^{+}} \frac{\partial g\left(\theta_{i}, \theta_{k}\right) / \partial \theta_{i}}{g\left(\theta_{i}, \theta_{k}\right)} d \theta_{i}\right]$. Therefore (7) holds if and only if $\frac{\partial g\left(\theta_{i}, \theta_{k}\right) / \partial \theta_{i}}{g\left(\theta_{i}, \theta_{k}\right)}$ is nondecreasing in $\theta_{k}$ which is in turn necessary and sufficient for $\frac{f_{a_{i}}\left(x_{i}, x_{k} ; a_{i}, a_{k}\right)}{f\left(x_{i}, x_{k} ; a_{i}, a_{k}\right)}$ to be nonincreasing in $x_{k}$, $i, k=A, B, i \neq k$

Each director should be then compensated in direct relation to his own outcome and in negative relation to the performance of the director of the other plant. Given that the random variables affecting the two outcomes are affiliated, the result of director $k$ is used as a signal of the realization of the random variable affecting $i^{\prime}$ s output. Since a high realization of $x_{k}$ (given $a_{k}$ ) signals a high realization of $\theta_{k}$ and since affiliation implies that a high $\theta_{k}$ is likely to be associated to a high $\theta_{i}$, a high level of $x_{k}$ can be interpreted as a signal of a relatively high $\theta_{i}$. This speaks in favor of luck, in the sense of exogenously good market conditions, for director $i$ and for this reason the latter should be paid less (for any given level of $x_{i}$ ), the higher is $x_{k}$. As pointed out by Holmström (1982) and Mookherjee (1984), whenever outputs are dependent, relative performance evaluation allows to filter out the common element of uncertainty and thus to improve upon independent compensations. These authors however do not fully characterize the compensation schedule $y_{i}(x)$. As it happens for the relation of $i^{\prime}$ s compensation to $x_{i}$ in the absence of the MLR assumption, the relation of $y_{i}(x)$ to $x_{k}$ could in principle take any form. Affiliation ensures that the compensation of an agent is monotonically nonincreasing in the performance of his peer.

\footnotetext{
${ }^{5}$ Affiliation implies that the covariance is non-negative while the reverse is not true (Tong, 1980). Note that independent variables are affiliated since in that case (7) always holds as an equality.
} 


\section{Concluding remarks}

We have studied the form of optimal incentives for the directors of two plants belonging to the same owner and selling the same good on geographically differentiated markets. If the demands on the two markets are stochastically dependent, the performance of one director gives some information about the realization of the random variable affecting the outcome of his colleague. This principal multi-agent model can thus be regarded as a kind of multi-signal model. We have shown, however, that fewer assumptions are needed than in the general multi-signal model (Sinclair-Desgagné, 1994) in order to provide appropriate extensions of the MLR and CDF conditions that justify the first-order approach in the single agent case. We have then investigated the viability of relative performance evaluation and we have shown that the compensation of one director is monotonically nonincreasing in the performance of the other if and only if the random variables are affiliated.

Clearly our results apply to other principal multi-agent settings. We could have considered compensation schedules of sellers working for the same firm or also those of CEOs of different firms as long as the outcome of each individual is known to all concerned. Alternatively, we could also have considered different workers or profit/cost centers inside a single firm. Whenever the uncertainty affecting the outcomes of different units or individuals can be represented by affiliated random variables, the optimal compensation of each unit/individual is a monotonically negative function of the performance of the others. What is crucial to our result is that separable signals of the performance of different agents are available so that, given the effort of an agent, his outcome can be interpreted as a signal of the random variable affecting his production. In our case this is guaranteed by separability of production functions. In general, production externalities or team production could also be considered as long as either a signal of each agent's performance is observable or the externalities are sufficiently small.

\section{References}

Antle, R. and A. Smith: An Empirical Investigation of the Relative Performance Evaluation of Corporate Executives, Journal of Accounting Research 24, 1-39 (1986).

Gibbons R. and K.J. Murphy: Relative Performance Evaluation for Chief Executive Officers, Industrial and Labor Relation Review 43, (1990).

Holmström, B.: Moral Hazard in Teams, Bell Journal of Economics 13, 324-40 (1982).

Holmström, B. and P. Milgrom: Regulating Trade Among Agents, Journal of Institutional and Theoretical Economics 146, 85-105 (1990).

Kren, L.: Common Uncertainty Effects on the Use of Relative Performance Evaluation for Corporate Chief Executives, Advances in Accounting 19, 119-138 (2002). 
Itoh, H.: Coalitions, Incentives and Risk Sharing, Journal of Economic Theory 60, 410-427 (1993).

Janakiraman S.N., Lambert, R.A. and F.D. Larcker: An Empirical Investigation of the Relative Performance Evaluation Hypothesis, Journal of Accounting Research 30, 53-69 (1992).

Jewitt, I.: Justifying the First-Order Approach to Principal-Agent Problems, Econometrica 56, 1177-1190 (1988).

Milgrom, P.R.: Good News and Bad News: Representation Theorems and Applications, Bell Journal of Economics 12, 380-391 (1981).

Milgrom, P.R. and R.J. Weber: A Theory of Auctions and Competitive Bidding, Econometrica 50, 1089-1122 (1982).

Mirrlees, J.A.: Notes on Welfare Economics, Information and Uncertainty, in Balch M.S., McFadden D.L. and S.Y. Wu (eds.) Essays on Economic Behavior and Uncertainty, Amsterdam: North-Holland 1974.

Mirrlees, J.A.: The Implications of Moral Hazard for Optimal Insurance, Seminar given at Conference in honour of Karl Borch, Bergen, mimeo, 1979.

Mookherjee, D.: Optimal Incentive Schemes with Many Agents, Review of Economic Studies 51, 433-446 (1984).

Rogerson, W.P.: The First-Order Approach to Principal-Agent Problems, Econometrica, 53, 1357-1368 (1985).

Sinclair-Desgagné, B.: The First-Order Approach to Multi-Signal PrincipalAgent Problems, Econometrica 62, 459-465 (1994).

Tong, Y.L.: Probability Inequalities in Multivariate Distributions, New York: Academic Press 1980. 